\title{
On the Uniqueness of String Theory
}

\section{Citation}

BERKOVITS, NATHAN, and CUMRUN VAFA. 1994. "ON THE UNIQUENESS OF STRING THEORY." Modern Physics Letters A 9 (7): 653-63. https://doi.org/10.1142/s0217732394003889.

\section{Permanent link}

http://nrs.harvard.edu/urn-3:HUL.InstRepos:41385044

\section{Terms of Use}

This article was downloaded from Harvard University's DASH repository, and is made available under the terms and conditions applicable to Other Posted Material, as set forth at http:// nrs.harvard.edu/urn-3:HUL.InstRepos:dash.current.terms-of-use\#LAA

\section{Share Your Story}

The Harvard community has made this article openly available.

Please share how this access benefits you. Submit a story.

Accessibility 
HUTP-93/A031, KCL-TH-93-13

\title{
On the Uniqueness of String Theory
}

\author{
Nathan Berkovits \\ Math Dept., King's College \\ Strand, London, WC2R 2LS, United Kingdom \\ Cumrun Vafa \\ Lyman Laboratory of Physics, Harvard University \\ Cambridge, MA 02138, USA
}

\begin{abstract}
We show that bosonic strings may be viewed as a particular class of vacua for $N=1$ superstrings, and $N=1$ superstrings may be viewed as a particular class of vacua for $N=2$ strings. Continuing this line of string hierarchies, we are led to search for a universal string theory which includes all the rest as a special vacuum selection.
\end{abstract}

$10 / 93$ 
One of the most beautiful aspects of string theory is that it has almost no adjustable parameters. The main choice to be made is the selection of a string vacuum. The only nonunique feature seems to be the selection of which worldsheet symmetries we are gauging. If we take the worldsheet gravity theory to be pure gravity $(\mathrm{N}=0)$, we get the bosonic strings, for $N=1$ supergravity we get the fermionic string, and for $N=2$ supergravity we get the $N=2$ strings. For closed strings, we can also choose heterotic combinations $(p, q)$ depending on which symmetries we choose for the left- or right-moving degrees of freedom (the most well known example being the $(0,1)$ heterotic string). In this paper we show that the choice of which string we consider may also be viewed just as another choice of string vacuum. In particular we will show that any string vacuum for a $(p, q)$ string can be viewed as a special choice of the string vacuum for $\left(p^{\prime}, q^{\prime}\right)$ string with $0 \leq p \leq p^{\prime} \leq 2$ and $0 \leq q \leq q^{\prime} \leq 2$. In other words we have a hierarchy $N=0 \subset N=1 \subset N=2$ and so in this class, the $(2,2)$ strings is the most general string which includes all the others as special choices of vacua.

When we speak of a $(p, q)$ string vacuum we mean a conformal theory with $(p, q)$ superconformal symmetry with appropriate central charged: $(N=0 \rightarrow c=26 ; N=1 \rightarrow$ $\hat{c}=10 ; N=2 \rightarrow \hat{c}=2$ ), where the last $\hat{c}=2$ corresponds to counting complex dimensions. What we shall say below for the left-movers can also be said for the right-movers, so in the following we will limit our discussion to the left-movers.

\section{1. $N=0 \subset N=1$}

Consider a bosonic string vacuum, i.e. a conformal theory with $c=26$. It is known [1] that if we use a $U(1)$ current of the matter system, there is a hidden $N=2$ supersymmetry in the combined matter-ghost system. However we have to assume the existence of a current in the matter system. In particular, choosing such a current in the case of strings on $R^{26}$ would break Lorentz invariance. However it turns out that if we slightly change the spin content of the ghost system (roughly speaking 'untwist it') without choosing any $U(1)$ currents, there is an $N=1$ supersymmetry for the combined matter and shifted

1 One can attempt some generalizations of the allowed vacua but we will not consider them here. In particular for strings with $c<1$ where the ghost system is mixed with the matter for a generic perturbation, we will have to broaden this strict definition of the vacuum. We expect that the hierarchy uncovered in this paper continues to hold even after we broaden the definition of the vacuum for each string theory. In this paper we limit ourselves to the case that all the cohomology comes from the matter sector with no mixing with the ghost. 
spin $(b, c)$ ghost system. Let us call the shifted spin ghost system by $\left(b_{1}, c_{1}\right)$ which has spin $(3 / 2,-1 / 2)$. Then denoting the energy momentum tensor for the matter system as $T_{m}$ (with central charge 26), we can write the generator of the $N=1$ superconformal alegebra as

$$
\begin{gathered}
G=b_{1}+c_{1}\left(T_{m}+\partial c_{1} b_{1}\right)+\frac{5}{2} \partial^{2} c_{1} \\
T=T_{m}-\frac{3}{2} b_{1} \partial c_{1}-\frac{1}{2} \partial b_{1} c_{1}+\frac{1}{2} \partial^{2}\left(c_{1} \partial c_{1}\right) .
\end{gathered}
$$

We have the following OPE:

$$
\begin{gathered}
G(z) G(0) \sim \frac{10}{z^{3}}+\frac{2 T}{z} \\
T(z) T(0) \sim \frac{15}{2 z^{4}}+\frac{2 T}{z^{2}}+\frac{\partial T}{z}
\end{gathered}
$$

which is an $N=1$ superconformal algebra with $\hat{c}=10(c=15)$. Note that the energymomentum tensor is the sum of the energy momentum tensor of the matter system and the spin $(3 / 2,-1 / 2)$-system $\left(b_{1}, c_{1}\right)$, with an extra 'improvement' term which can be written as $\partial J / 2$ where $J=\partial\left(c_{1} \partial c_{1}\right)$. Note that since $J J \sim 0$, it does not affect the central charge of the system. In the path-integral language, this is the same as adding a term proportional to $\int R\left(c_{1} \partial c_{1}\right)$ to the action. Since this term violates $c_{1} b_{1}$ number by 2 units, it will not contribute to correlations that we shall consider below due to $c_{1} b_{1}$ ghost number conservation.

The existence of an $N=1$ superconformal algebra in the bosonic string after shifting the spins of the ghosts is non-trivial and is not the consequence of the $N=2$ superconformal symmetry considered in $[1]^{2}$. However the form of $G$ is reminiscent of $G^{-}+G^{+}$from that reference where $G^{-}=b$ and $G^{+}=j_{B R S T}$ (up to a total derivative term). Note also that for $G$ to have spin $3 / 2$ we needed to shift the spins of $\left(b_{1}, c_{1}\right)$ to be $(3 / 2,-1 / 2)$.

Thus we see that the combined system $\left(T_{m},\left(b_{1}, c_{1}\right)\right)$ has $N=1$ superconformal symmetry with $\hat{c}=10$ which thus makes it a viable candidate for the matter system of an $N=1$ fermionic string. We will now show the equivalence

$$
\left(T_{m},\left(b_{1}, c_{1}\right) ;(b, c),(\beta, \gamma)\right) \rightarrow\left(T_{m} ;(b, c)\right)
$$

where the lefthand side refers to an $N=1$ vacuum with $((b, c),(\beta, \gamma))$ denoting the superdiffeomorphism ghosts. Formally it seems plausible that this correspondence might

2 In particular the map considered there takes the critical bosonic string on $R^{26}$ to the $\mathrm{N}=2$ conformal theory with $\hat{c}=3(c=9)$. 
work since if we ignore the zero modes of the fields, on the lefthand side the fermionic $\left(b_{1}, c_{1}\right)$ system cancels the bosonic ghost $(\beta, \gamma)$ because they have the same spin but opposite statistics. We are thus left with $T_{m}$ and the $(b, c)$ ghosts, which is all that we have on the righthand side. Before going on to show how the zero modes work, let us discuss the physical fields of the resulting string theory and the computation of amplitudes at the tree level.

Bosonic string states are described by dimension 1 vertex operators, $V$, which will be assumed to be constructed entirely out of the matter fields in $T_{m}$. Although $c_{1} V$ commutes with the BRST charge of the bosonic string, it is a dimension $1 / 2$ operator after shifting the spin of the $c_{1}$ field. The corresponding BRST-invariant operator for the $\mathrm{N}=1$ string is therefore $c e^{-\phi} c_{1} V$ (this is $N=1 \mathrm{BRST}$ invariant since $c_{1} V$ is a primary of the $N=1$ superconformal matter system with the appropriate dimension). In $\mathrm{N}=1$ language, this operator is in the ghost-number 0 picture and to get other pictures, one attaches the picture-changing operator

$$
Z=\left\{Q_{N=1}, \xi\right\}=e^{\phi} G+2 \partial \eta e^{2 \phi} b+\eta \partial\left(e^{2 \phi} b\right)+c \partial \xi
$$

where $G$ is defined in equation $(1)$ and the $(\xi, \eta, \phi)$ fields come from bosonizing the $(\beta, \gamma)$ ghosts in the usual way. For example, the BRST-invariant operator in the ghost-number 1 picture is $c V+\gamma c_{1} V$. The integrated form of these operators is obtained in the usual way by commuting with $\int b$ which leads to $\int V$.

For n-point amplitudes on a sphere, the relevant correlation function is therefore:

$$
\left\langle\left(c e^{-\phi} c_{1} V_{1}\right)\left(c e^{-\phi} c_{1} V_{2}\right)\left(c V_{3}+\gamma c_{1} V_{3}\right) \int V_{4} \ldots \int V_{n}\right\rangle
$$

Note that the second part of the vertex operator for $V_{3}$ does not contribute due to $b_{1} c_{1}$ conservation (as mentioned earlier, this conservation also allows us to ignore the 'improvement' term $\partial\left(c_{1} \partial c_{1}\right)$ that appeared in $T$ of equation (1)). After taking into account the extra $c_{1} e^{-\phi}$ that appear in the first two vertex operators, it is easily checked that the path integral over the $\left(b_{1}, c_{1}\right)$ fields precisely cancels the path integral over the $(\beta, \gamma)$, including the zero modes, leaving us with the usual bosonic string correlation function for the tree amplitude.

For surfaces of genus $\mathrm{g}$, the relevant $\mathrm{N}=1$ correlation function is

$$
\left\langle\prod_{j=1}^{3 g-3} \int d y_{j} \mu_{j}\left(y_{j}\right) b\left(y_{j}\right) \quad \prod_{k=1}^{2 g-2} Z\left(u_{k}\right) \quad \int V_{1} \ldots \int V_{n}\right\rangle
$$


where $Z$ is the picture-changing operator defined in equation (3) and $\mu_{j}$ comes from the usual $3 g-3$ Beltrami differentials. By $b_{1} c_{1}$ conservation, only the $e^{\phi} b_{1}$ term from $Z$ contributes to the correlation function (the other terms in $Z$ all have non-positive $b_{1} c_{1}$ number and the total $b_{1} c_{1}$ number must equal $\left.2 g-2\right)$. Since $e^{\phi} b_{1}=\delta(\beta) b_{1}$ [2], the path integral over the $\left(b_{1}, c_{1}\right)$ fields again precisely cancels the path integral over the $(\beta, \gamma)$ fields, including the zero modes, and we are left with the usual bosonic string correlation function on a genus $g$ surface. Note that this correlation function is independent both of the position of the $Z$ 's and of the spin structure chosen for the fields with half-integer spin.

However there is a point that we need to be more careful about. In the superstring theory, we are instructed to sum over all spin structures and divide the resulting amplitude by $2^{g}$. We have just argued that for each spin structure, the resulting amplitude is the same as that for the bosonic string. Therefore summing over all spin structures would give us a factor

$$
\left[\frac{1}{2^{g}} \sum_{\text {spin }} 1\right] Z_{\text {structures }}=\frac{2^{2 g}}{2^{g}} Z_{\text {bosonic }}=2^{g} Z_{\text {bosonic }}
$$

But this is in contradiction with the unitarity of the fermionic string. We know that the bosonic string is unitary, and moreover, the only way to modify the genus $g$ partition function consistent with unitarity is to let $Z_{g} \rightarrow \lambda^{g-1} Z_{g}$. But the above factor is $2^{g}$ and not $2^{g-1}$, which means that we cannot absorb it into a redefinition of the coupling constant. The resolution of this puzzle follows from the fact that we know only that the norm of the contribution from each spin structure is the same as that of the bosonic string. We cannot argue about its phase. However invariance under modular transformations (which mix even and odd spin-structures seperately) implies that we have essentially only two choices. Let us assume that all the even spin structures come with a factor of $(+1)$ and all the odd ones with a factor of $(-1)$. We thus see that the computation of the $N=1$ side leads to

$$
\frac{1}{2^{g}}\left[\sum_{\text {spin struct. }}(-1)^{\sigma}\right] Z_{\text {bosonic }}=\frac{1}{2^{g}}\left[2^{g-1}\left(2^{g}+1\right)-2^{g-1}\left(2^{g}-1\right)\right] Z_{\text {bosonic }}=Z_{\text {bosonic }}
$$

This is exactly what we wished to have with no adjustments of coupling constants! So it is crucial for the consistency of our equivalence that the difference between the number of even and odd spin structure in genus $g$ be $2^{g}$, which magically enough is the case!

If we apply this computation to the genus 1 case, what we are saying is that instead of getting $\frac{1}{2}(1+1+1+1)=2$ times the bosonic string amplitude, we get the three even spin 
structures contributing $\mathrm{a}+1$ and the odd one contributing a $(-1): \frac{1}{2}(1+1+1-1)=1$. Translating this to the language of the Hilbert space, we see that the Ramond sector does not contribute any physical states for this vacuum because all the states in it are GSO odd! So all the physical states come from the NS sector and they are in one to one correspondence with the bosonic string states.

This concludes showing that we may imbed the $N=0$ string in the $N=1$ string, albeit as a very particular subclass of $N=1$ vacua.

2. $N=1 \subset N=2$

We can now proceed by analogy to imbed the $N=1$ string in the $N=2$ string. The idea is to start with an $N=1 \mathrm{SCFT}$ with $\hat{c}=10$, add the usual $N=1$ ghosts, then shift the spins of the ghosts down by $1 / 2$ and try to construct out of this total system an $N=2$ SCFT with $\hat{c}=2$ (where $\hat{c}=2$ refers to the $N=2$ central charge, i.e., it corresponds to $c=6$ ). Then we should take this system as the matter system for the $N=2$ strings, add the $N=2$ ghost system, and show that the computations of amplitudes in the $N=2$ formalism with this matter system is the same as in the original $N=1 \mathrm{SCFT}$ coupled to $N=1$ supergravity.

Let us represent the $N=1$ matter system with $\hat{c}=10$ by $\left(T_{m}, G_{m}\right)$, i.e. by its energy momentum tensor and by its supercurrent. Let us denote the spin-shifted ghost system by fermionic $\left(b_{1}, c_{1}\right)$ of spin $(3 / 2,-1 / 2)$ and bosonic $\left(\beta_{1}, \gamma_{1}\right)$ of spin $(1,0)$. Then the claim is that this system magically has an $N=2$ superconformal symmetry with $\hat{c}=2$. This same $N=2$ algebra was constructed in [3] in showing that $N=1$ NSR strings can be mapped to GS strings in the $N=2$ formulation. In the following we will sometimes find it convenient to use the bosonized version of the $\left(\beta_{1}, \gamma_{1}\right)$ system, in which case we write

$$
\gamma_{1}=\eta_{1} \exp (\phi) \quad \beta_{1}=\partial \xi_{1} \exp (-\phi)
$$

where $\phi$ is the usual bosonized ghost field of the fermionic string [4] with appropriate background charge and the $\left(\eta_{1}, \xi_{1}\right)$ are spin-shifted to $(3 / 2,-1 / 2)$ in order to reproduce correctly the spin-shifted $\left(\beta_{1}, \gamma_{1}\right)$ system (or more appropriately, we could have started with the bosonized version of this system as the fields to be added to the matter system). Now we are ready to write the $N=2$ generators of the combined system $\left(\left(T_{m}, G_{m}\right),\left(b_{1}, c_{1}\right),\left(\beta_{1}, \gamma_{1}\right)\right)$ :

$$
G^{-}=b_{1}
$$




$$
\begin{gathered}
G^{+}=\gamma_{1} G_{m}+c_{1}\left(T_{m}-\frac{3}{2} \beta_{1} \partial \gamma_{1}-\frac{1}{2} \partial \beta_{1} \gamma_{1}\right)-\gamma_{1}^{2} b_{1}+\partial\left(c_{1} \xi_{1} \eta_{1}\right)+\partial^{2} c_{1}+b_{1} c_{1} \partial c_{1} \\
T=T_{m}-\frac{3}{2} \beta_{1} \partial \gamma_{1}-\frac{1}{2} \partial \beta_{1} \gamma_{1}-b_{1} \partial c_{1}-\frac{1}{2} \partial\left(b_{1} c_{1}-\xi_{1} \eta_{1}\right) \\
J=b_{1} c_{1}+\xi_{1} \eta_{1}
\end{gathered}
$$

It is tedious but a straightforward exercise to show that these form an $N=2$ superconfomal system with $\hat{c}=2$, which is the critical value needed for the $\mathrm{N}=2$ string. Note in particular that we could not write the $N=2$ algebra in a simple form just in terms of the $\beta_{1}, \gamma_{1}$ system because of the appearance of $\xi_{1} \eta_{1}$ in the currents. Furthermore, it does not seem possible to enlarge this algebra to the $\mathrm{N}=3$ algebra found in reference [1] since $\beta_{1}$ no longer has the right OPE with $J$ to be an $S O(3)$ current.

We now take this matter system and couple it to $N=2$ supergravity. So the total system including the ghosts is

$$
\left(\left(T_{m}, G_{m}\right),\left(b_{1}, c_{1}\right),\left(\eta_{1}, \xi_{1}\right), \phi ;(b, c),\left(\beta^{ \pm}, \gamma^{ \pm}\right),(\eta, \xi)\right)
$$

where $(b, c)$ are the fermionic diffeomorphism ghosts of spin $(2,-1),\left(\beta^{ \pm} \gamma^{ \pm}\right)$are the bosonic superdiffeomorphism ghosts of spin $(3 / 2,-1 / 2)$, and $(\eta, \xi)$ are the fermionic $U(1)$ diffeomorphism ghosts of spin $(1,0)$. We will now show that scattering amplitudes calculated using this $\mathrm{N}=2$ string theory agree with amplitudes calculated using the original $\left(T_{m}, G_{m}\right)$ system coupled to $\mathrm{N}=1$ supergravity.

First let us argue how the non-zero modes work as before: Consider an arbitrary $N=2$ moduli, which consists of a worldsheet moduli and the choice of a flat $U(1)$ connection. Note that the only $U(1)$ charged fields are $\left(\left(b_{1}, c_{1}\right),\left(\eta_{1}, \xi_{1}\right) ;\left(\beta^{ \pm}, \gamma^{ \pm}\right)\right)$which all have spin $(3 / 2,-1 / 2)$, and the first two are fermionic while the last two are bosonic. Thus the non-zero modes of all of these fields cancel out and we are left with

$$
\left(\left(T_{m}, G_{m}\right), \phi ;(b, c),(\eta, \xi)\right)
$$

which is precisely the matter and ghost content of the $N=1$ string (when we think of the leftover $(\phi, \eta, \xi)$ as the bosonized $(\beta, \gamma)$ ghost). Before describing how the zero modes work, let us construct the physical vertex operators of the resulting $N=2$ string theory and compute the tree level amplitudes.

In $\mathrm{N}=1$ language, physical states are described by vertex operators in different pictures. For example, Neveu-Schwarz states can be described by the operator $c_{1} e^{-\phi} V$ in the 
ghost-number 0 picture or by the operator $c_{1}\left[G_{m}, V\right]+\gamma_{1} V$ in the ghost-number 1 picture. In the above expressions, $V$ is assumed to be a dimension $1 / 2$ operator constructed entirely out of $\mathrm{N}=1$ matter fields and $\left[G_{m}, V\right]$ means the $z^{-1}$ piece from the OPE of $G_{m}$ with $V$. Similarly, Ramond states can be described by the operator $c_{1} e^{-\phi / 2} W$ in the ghost-number $1 / 2$ picture or by $c_{1} e^{+\phi / 2}\left[G_{m}, W\right]+\left(b_{1} c_{1} \eta_{1}+2 \partial \phi \eta_{1}+2 \partial \eta_{1}\right) e^{3 \phi / 2} W$ in the ghost-number $3 / 2$ picture, where $W$ is a dimension $5 / 8$ operator constructed entirely out of $\mathrm{N}=1$ matter fields and $\left[G_{m}, W\right]$ means the $z^{-1 / 2}$ piece from the OPE of $G_{m}$ with $W$.

In $\mathrm{N}=2$ language, there are not only two picture-changing operators defined by

$$
Z^{ \pm}=\left[Q_{N=2}, \xi^{ \pm}\right]=e^{\phi^{\mp}}\left[G^{ \pm}+(b \mp 1 / 2 \partial \eta) \gamma^{ \pm}-\eta \partial \gamma^{ \pm}\right]+c \partial \xi^{ \pm}
$$

but also instanton-number-changing operators 3 defined by

$$
I=e^{\int J+\phi^{+}-\phi^{-}}=b_{1} \xi_{1} e^{\phi^{+}-\phi^{-}} \quad \text { and } \quad I^{-1}=e^{-\left(\int J+\phi^{+}-\phi^{-}\right)}=c_{1} \eta_{1} e^{\phi^{-}-\phi^{+}}
$$

where $G^{ \pm}$and $J$ are defined in equation (5) . It is easy to verify that these operators are in the $\mathrm{N}=2$ BRST cohomology but their derivatives are BRST trivial, so they can be used to express the same physical state in terms of different $\mathrm{N}=2$ vertex operators. Note that unlike in the $\mathrm{N}=1$ formalism, these different vertex operators may contain the same $\mathrm{N}=2$ ghost number. [5]

For example, a physical Neveu-Schwarz state with $\mathrm{N}=2$ ghost number -1 can be described by the BRST-invariant vertex operator $c e^{-\phi^{+}-\phi^{-}} \xi_{1} c_{1} e^{-\phi} V$. A BRST-invariant ghost-number +1 vertex operator can be constructed by attaching $Z^{-} Z^{-} I^{-1}$, which results in the operator $c e^{-\phi} V+\gamma^{-} c_{1} e^{-\phi} V$. Alternatively, one could attach $Z^{+} Z^{-}$, which results in the BRST-invariant ghost-number +1 vertex operator $c[G, V]+c \partial\left(c_{1} \xi_{1} e^{-\phi} V\right)+\gamma^{-} \xi_{1} e^{-\phi} V$.

Similarly, a physical Ramond state with $\mathrm{N}=2$ ghost-number 0 is described by the vertex operator $c e^{-\phi^{+}} c_{1} e^{-\phi / 2} W$. Ghost-number +1 operators can be obtained by attaching $Z^{-}$to get $c e^{-\phi / 2} W+\gamma^{-} c_{1} e^{-\phi / 2} W$, or by attaching $Z^{+} I$ to get $c\left(e^{\phi / 2}[G, W]+\eta_{1} b_{1} e^{3 \phi / 2} W+\right.$ $\left.\partial\left(c_{1} \xi_{1} e^{-\phi / 2} W\right)\right)+\gamma^{-} \xi_{1} e^{-\phi / 2} W$.

These vertex operators can be expressed in integrated form by commuting with $\int b$, and it is easy to show that in this form, they precisely coincide with the integrated form of

3 The instanton charge is defined as $N_{I}=\int\left(\partial \phi-\eta_{1} \xi_{1}\right)$ which is $\mathrm{N}=2$ BRST invariant and satisfies $\left[N_{I}, I^{ \pm}\right]= \pm I^{ \pm}$. The instanton number, $n_{I}$, of an operator $V$ is defined by $\left[N_{I}, V\right]=n_{I} V$. If $V$ is $\mathrm{U}(1)$-invariant and is constructed entirely out of $\mathrm{N}=2$ matter fields, $n_{I}$ is equal to the $\mathrm{N}=1$ ghost number since $N_{I}=\int\left(c_{1} b_{1}+\partial \phi+J\right)$. 
the $\mathrm{N}=1$ expressions. For example, the $\mathrm{N}=2$ Neveu-Schwarz vertex operators take the form $\int e^{-\phi} V$ and $\int[G, V]$, while the $\mathrm{N}=2$ Ramond vertex operators take the form $\int e^{-\phi / 2} W$ and $\int\left(e^{\phi / 2}[G, W]+e^{3 \phi / 2} \eta_{1} b_{1} W\right)$. The equivalence of $\mathrm{N}=1$ and $\mathrm{N}=2$ vertex operators in integrated form is not so surprising since $\left[Q_{N=1}, V\right]=0$ and $\left[b_{1}, V\right]=0$ is almost enough to imply that $\left[Q_{N=2}, V\right]=0$. The only obstacle may come from singularities which are total derivatives in the OPE of $G^{+}$with $V$ (these singularities would spoil the N=2 BRST invariance of $V$, but not the N=1 BRST invariance). Singularities of this type do not occur in the pictures considered above, however they probably do occur for vertex operators in other pictures.

To calculate tree amplitudes with $m$ bosons and $2 n$ fermions in the $\mathrm{N}=2$ formalism, one needs to evaluate the correlation function

$$
\begin{gathered}
\left\langle\xi\left(z_{0}\right)\left(c e^{-\phi^{+}-\phi^{-}} \xi_{1} c_{1} e^{-\phi} V_{1}\right)\left(c e^{-\phi^{+}-\phi^{-}} \xi_{1} c_{1} e^{-\phi} V_{2}\right)\right. \\
\left.\left(c e^{-\phi / 2} W_{1}+\gamma^{-} c_{1} e^{-\phi / 2} W_{1}\right) \int e^{-\phi / 2} W_{2} \ldots \int V^{-\phi / 2} W_{n}\right] \\
\left.\int\left(e^{\phi / 2}\left[G, W_{n+1}\right]+e^{3 \phi / 2} \eta_{1} b_{1} W_{n+1}\right) \ldots \int\left(e^{\phi / 2}\left[G, W_{2 n}\right]+e^{3 \phi / 2} \eta_{1} b_{1} W_{2 n}\right)\right\rangle
\end{gathered}
$$

where the zero mode of the $\xi$ ghost needs to be inserted in order to get a non-zero amplitude (the correlation function is independent of the location of the insertion). Because of $e^{\phi}$ conservation, the second term in the vertex operators $W_{n+1}$ to $W_{2 n}$ never contributes to the correlation function. Similarly, the second term in the vertex operator for $W_{1}$ does not contribute by $b c$ conservation. For these reasons, the $\left(b_{1}, c_{1}\right)$ and $\left(\xi_{1}, \eta_{1}\right)$ path integrals are easily shown to cancel the $\left(\beta^{ \pm}, \gamma^{\mp}\right)$ path integrals. The resulting correlation function is precisely the relevant one for the $\mathrm{N}=1$ calculation of the scattering amplitude (the second term in $W_{n+1}$ to $W_{2 n}$ can also be ignored in the $\mathrm{N}=1$ calculation because of $e^{\phi}$ conservation). Note that the ability to ignore the second term in $W_{n+1}$ to $W_{2 n}$ depended crucially on the pictures chosen for the other vertex operators, and for a general choice of pictures, it is not obvious how to prove directly that the $\mathrm{N}=1$ and $\mathrm{N}=2$ correlation functions coincide. Of course, they can be proven indirectly to coincide by using the fact that all pictures are related to each other by BRST-trivial operations, which only changes the integrand of the scattering amplitude by a total derivative.

To prove the equivalence for multiloop amplitudes, it is also useful to choose special pictures for the external states. For a genus $g$ surface, the simplest amplitude to compare contains $m$ Neveu-Schwarz states, all in the ghost-number 0 picture, and $4 g-4+2 n$ 
Ramond states, $n$ in the ghost-number $-1 / 2$ picture, and $4 g-4+n$ in the ghost-number $+1 / 2$ picture. With this choice of picture, there is no need in the $\mathrm{N}=1$ formalism to insert picture-changing operators at additional points.

In the $\mathrm{N}=2$ formalism, the relevant measure on the moduli space is:

$$
\begin{gathered}
\prod_{i=1}^{g} \int d m_{i}\left\langle\xi\left(z_{0}\right) \int d w_{i} \eta\left(w_{i}\right) \prod_{j=1}^{3 g-3} \int d y_{j} \mu_{j}\left(y_{j}\right) b\left(y_{j}\right) \prod_{k=1}^{4 g-4} Z^{-}\left(u_{k}\right)\right. \\
\int\left[G, V_{1}\right] \ldots \int\left[G, V_{m}\right] \int e^{-\phi / 2} W_{1} \ldots \int e^{-\phi / 2} W_{n} \\
\left.\int e^{\phi / 2}\left[G, W_{n+1}\right]+e^{3 \phi / 2} \eta_{1} b_{1} W_{n+1} \ldots \int e^{\phi / 2}\left[G, W_{4 g-4+2 n}\right]+e^{3 \phi / 2} \eta_{1} b_{1} W_{4 g-4+2 n}\right\rangle
\end{gathered}
$$

where $m_{i}$ are the complex $\mathrm{U}(1)$ moduli whose integration region is $C^{g} /\left(Z^{g}+\tau Z^{g}\right)$, $\int d w_{i} \eta\left(w_{i}\right)$ are the corresponding $U(1)$ ghost insertions, and the $4 g-4 Z^{-}$'s come from integrating over the supermoduli. Note that because the combined instanton number of the vertex operators is $2 g-2$ (it is equal to the $\mathrm{N}=1$ ghost number of the vertex operators), the surface itself must carry instanton number $2-2 g$ to get a non-zero amplitude. On such a surface, the conformal weight of all fields is shifted (if one thinks of identifying the spin connection with the $U(1)$ connection) by their $\mathrm{U}(1)$ charge (i.e. the screening charge for $\left[\int b_{1} c_{1}, \int \eta_{1} \xi_{1}, \phi^{+}, \phi^{-}\right]$is shifted from $[2,2,2,2]$ to $\left.[4,0,4,0]\right)$, and there are $4 g-4$ super-moduli for one of the two right-moving gravitinos and none for the other one.

As in the tree amplitude, the second term in the vertex operators for $W_{n+1}$ to $W_{4 g-4+2 n}$ can be ignored due to $e^{\phi}$ conservation, implying by $b_{1} c_{1}$ conservation that only the $e^{\phi^{+}} b_{1}$ part of $Z^{-}$contributes to the correlation function (because of the shift in screening charge, $4 g-4 b_{1}$ 's are needed for a non-zero result). Using these facts, it is again easy to show that the $\left(b_{1}, c_{1}\right)$ and $\left(\eta_{1}, \xi_{1}\right)$ path integrals precisely cancel the $\left(\beta^{\mp}, \gamma^{ \pm}\right)$ path integrals including the zero modes. Since all other fields contribute identically in the two formalisms, the integrands of the scattering amplitudes calculated using the $\mathrm{N}=1$ and $\mathrm{N}=2$ theory coincide 1 . Note that because the $\mathrm{N}=2$ matter system has not yet been GSO

4 It is important to study precisely how the left- and right-movers are put together (in particular the chiral one we are discussing here is naturally identified with the $Z_{\infty}$ string of [6]). A related issue is that the integration over the $U(1)$ Jacobian leaves us with a factor of $\operatorname{det} \tau_{2}$ which should be canceled by an inverse factor from the $\beta_{1}, \gamma_{1}$ part of the matter system as would be dictated by modular invariance. Another related issue is the fact that $\phi$ is a negative-energy boson which may introduce unphysical poles, as is familiar from the $N=1$ superstrings. Similar issues arise and have been dealt with in the context of the $\mathrm{N}=2$ formulation of the Green-Schwarz superstring [5]. 
projected, one still needs to sum over spin structures in the $\mathrm{N}=2$ matter fields in order to recover the complete $\mathrm{N}=1$ scattering amplitude.

If a different picture is chosen or if less than $4 g-4$ Ramond states are present, it is still expected by unitarity arguments that the scattering amplitudes coincide, however it is probably not true that the integrands of the amplitudes coincide (they only need to agree up to total derivatives in the moduli). This is not surprising since although the integrands are required by unitarity to coincide in light-cone gauge, gauge transformations away from light-cone gauge may shift the integrands by total derivatives. Since there is no simple relation between an $\mathrm{N}=1$ gauge transformation and an $\mathrm{N}=2$ gauge transformation ( $G$ of $\mathrm{N}=1$ is related in a complicated way to $G^{ \pm}$of $\mathrm{N}=2$ ), there is no reason why the shift of the $\mathrm{N}=1$ integrand and the shift of the $\mathrm{N}=2$ integrand should be equivalent.

\section{Conclusions}

We have seen that strings are more unique than had been suspected. The most general string studied so far, which includes all the other ones as special cases is the $N=2$ string. It is rather amusing that the more conventional choice for the vacuum of the $N=2$ string was also discovered to have magical properties and in particular be related to self-dual geometries in four dimensions [6], and was conjectured to be the master theory for all integrable models (see [7] for a review of the literature on $N=2$ strings).

However it is natural to ask if the story stops here. In particular, it has been recently found that the $N=2$ string has a hidden new $N=4$ superconformal symmetry [8] which suggests that this chain of construction can be continued at least one more step to the $N=4$ strings which magically enough has critical dimension zero. Since the $N=4$ strings have not been studied in depth, this remains conjectural but we find it aesthetically compelling for the chain of hierarchy to continue to the $N=4$ case.

There may be other directions in which one can generalize these constructions: We have seen that there is a hierarchy of strings, depending on which features of moduli space of Riemann surfaces we decide to focus on. The more 'general' string theory is the one which allows the partition function to depend non-trivially on more extra data available. For example, we have seen that special vacua of $N=1$ strings that do not depend on spin structure reduce to the $N=0$ strings. In this sense it is easy to understand why $N=0$ strings should be viewed as a special case of $N=1$ strings and not the other way around. Similarly, special $N=2$ string vacua which are insensitive to the $U(1)$ moduli of $N=2$ reduce to the $N=1$ string. Therefore we are naturally led to ask what other 
structures can we put on a Riemann surface? One such direction could be $W_{N}$ strings (see [9] for a review of recent results), or following the notion that the most structured string is the most general one, we could go on to $W_{\infty}$ strings (or some supersymmetric generalization thereof). It is quite amusing in this connection that in [6] it was noted that the 'most symmetrical' choice for a target space of $N=2$ strings possesses $W_{\infty}$ symmetry in the target space which by worldsheet-target duality was conjectured to transmute to the worldsheet. It would be interesting to pursue such directions more seriously and see if we are close to identifying the most general string theory.

At any rate, the search for the universal string theory (UST), the one which includes all the others by special choices of vacua, is now on! One would naturally expect that string symmetries are most manifest in such a universal theory. The world around us has decided to choose a highly asymmetrical vacuum of this very symmetrical string, maybe in the form of the heterotic string. But, as is the case with spontaneously broken symmetries, we expect that for deepest insights into a theory we have to understand the most symmetrical formulation of it and not concentrate on the 'asymmetrical' string vacuum! In this sense, more or less realistic string vacua such as the heterotic string play the role of a 'symmetry broken phase' of the UST.

We would like to thank the hospitality of Rutgers University where this project was initiated. We would also like to thank Michael Bershadsky, Mike Douglas, Mike Freeman, Martin Rocek, Nathan Seiberg, Warren Siegel, and Peter West for useful discussions. N.B. would also like to thank Harvard University for its hospitality, and the SERC for financial support. The research of C.V. is supported in part by a Packard fellowship and NSF grants PHY-87-14654 and PHY-89-57162. 


\section{References}

[1] B. Gato-Rivera and A.M. Semikhatov, Phys. Lett. B293 (1992) 72-80;

M. Bershadsky, W. Lerche, D. Nemeschansky, N.P. Warner, Extended N=2 Superconformal Structure of Gravity and W Gravity Coupled to Matter CERN-TH-6694-92, hep-th/9211040.

[2] E.Verlinde and H.Verlinde, Phys. Lett. B192 (1987) 95.

[3] N. Berkovits, The Ten Dimensional Green-Schwarz Superstring is a Twisted NeveuSchwarz-Ramond String, hep-th/9308129.

[4] D. Friedan, E. Martinec and S. Shenker, Nucl. Phys. B271 (1986) 93.

[5] N. Berkovits, Nucl. Phys. B395 (1993) 77, hep-th/9208035; Phys. Lett. B300 (1993) 53 and Finiteness and Unitarity of Lorentz-Covariant Green-Schwarz Superstring Amplitudes, KCL-TH-93-6, hep-th/9303122 (to appear in Nucl.Phys.B).

[6] H. Ooguri and C. Vafa, Nucl. Phys. B367 (1991)83 ; B361 (1991) 469; Mod. Phys. Lett. A5 (1990) 1389.

[7] N. Marcus, A Tour Through N=2 Strings, hep-th/9211059.

[8] A. Giveon and M. Rocek, On the BRST Operator Structure of the N=2 String, hepth/9302049;

J. Gomis and H. Suzuki, Phys. Lett. B278 (1992) 266.

[9] C.N. Pope, W-Strings 93, hep-th/9309125;

P. West, A Review of W Strings, hep-th/9309095, Goteborg-ITP-93-40. 